\title{
Multi-sensor Exercise-based Interactive Games for Fall Prevention and Rehabilitation
}

\author{
António Santos, Vânia Guimarães, Nuno Matos, João Cevada, Carlos Ferreira, Inês Sousa \\ Fraunhofer Portugal AICOS \\ Rua Alfredo Allen 455, 4200-135 Porto, Portugal \\ Email: \{antonio.santos, vania.guimaraes, nuno.matos, joao.cevada, carlos.ferreira, ines.sousa\}@ fraunhofer.pt
}

\begin{abstract}
According to statistics, one in every three adults ageing 65 or older falls every year. Every fall may lead to long-term consequences due to fractures or even neurological damages. These consequences have severe impact in their quality of life, independence and confidence, ultimately increasing the risk of early death. Moreover, the risk of falling increases as age advances. Fortunately, several studies reveal that specific exercise programmes may help in reducing the risk of falling if performed correctly and frequently. However, user engagement and adherence to these programmes are still low mainly due to motivational factors, since interventions are usually long, unadapted and unchallenging. In this paper, a new solution is presented, which uses the concept of interactive games using motion sensors to tackle low adherence (through gaming motivation) and help in physical rehabilitation and reduce fall risk on elderly people by improving balance, muscle strength and mobility. It is intended to be used in community or domestic unsupervised contexts and supports relatively inexpensive sensing equipment (currently Kinect ${ }^{\circledR}$, Leap Motion ${ }^{\circledR}$, Orbotix Sphero ${ }^{\circledR}$ and Smartphones) and common platforms (desktop and mobile). Tests were already undertaken with several individuals ageing 65 or more and the results were analysed and discussed, being generally positive, despite some issues in the movement detection algorithms.
\end{abstract}

Keywords_physical exercises; fall prevention; rehabilitation; exergames; elderly; natural user interfaces; kinect; smartphone

\section{INTRODUCTION}

Falls are dangerous and unfortunately common for older adults aged 65 years and older [1]. More than $30 \%$ of older people fall at least once a year [2], which may cause injury, suffering, fear, loss of independence and reduced quality of life. Therefore, falls have a significant socio-economic impact [3].

Falls can be due to a wide variety of causes, including poor balance, muscle weakness, limited mobility and slow reaction time [2], [4]. Additionally, these problems are considered to be among the reversible factors for which interventions have the greatest promise for preventing falls. Specific exercises for fall prevention have been proven extremely effective in improving balance, strength and mobility [4], offering an opportunity to reduce falls and fall-related injuries [5], [6].

Despite the clear evidence demonstrating the benefits of exercise in reducing fall risk, adherence to exercise programmes in fall prevention is often low [7], [8]. In fact, to be effective, these exercises have to be performed at least twice or three times a week [9], which means that a person needs to go to clinical environments where group sessions are conducted and/or perform the exercises at home. Due to the high costs of transportation, lack of motivation and confidence, seniors frequently decide not to exercise.

Similar problems are faced by victims of stroke or other neurological disorders with impact on mobility who generally have to undergo physiotherapy at clinics as part of their rehabilitation process. In this case, performing rehabilitation exercises at home can benefit patients, by providing them the opportunity to engage more often in therapy. The gamification of exercises may also contribute to a more compelling and frequent experience than repetitive traditional workouts, while also improving the cost-efficacy of recovery [10], [11].

One method by which compliance with exercise programmes can be improved involves the use of enjoyable exercise-based video games, which are often referred to as ExerGames. They combine movement with fun and entertainment, while they evaluate players' movements, providing feedback on game performance. ExerGames can also promote social interaction via competition or collaboration, which promotes motivation and adherence to exercise programmes [6], [8].

Existing ExerGames are usually available for specific platforms, which are proprietary solutions with closed implementation, featuring the support for a single type of sensor. Most of these games are not specific for fall prevention or rehabilitation, therefore neither exercises nor interfaces are adapted to these purposes and target groups [12]. Moreover, most fall prevention and balance training programmes which are set in clinical environments use equipment that most people cannot afford.

In this project, a multi-platform framework was designed and implemented for retrieving data from multiple standard motion-based sensor devices. Interactive games for exercise monitoring, fall prevention, physiotherapy and rehabilitation were developed taking into account the specificities of the exercises and their target groups, namely seniors and victims of neuromotor disorders.

In previous stages of development of this project, games focused on the rehabilitation of the upper limbs and hands in victims of neurological disorders (namely stroke) were developed and tested. These games were based on the aforementioned framework, and used Microsoft Kinect ${ }^{\circledR}$ [13], Leap Motion ${ }^{\circledR}[14]$ and Orbotix Sphero ${ }^{\circledR}$ [15] to track movements [16].

This paper describes the architecture of the system and 
the development of interactive games for fall prevention. Preliminary results of a usability study for these games are presented and discussed.

\section{BACKGROUND}

Scientific and health promotion literature is showing an increasing interest in methods for reducing fall risk factors and falls. Specific physical exercises have been proven effective in improving muscle strength, flexibility, balance and reaction time in any age, offering an opportunity to reduce falls and fall-related injuries [9], [17].

Several exercise programmes for fall prevention in older adults have been proposed, following a number of requirements previously established for this kind of exercises and target group. The Otago Exercise Programme, for example, has been shown to reduce falls by over $30 \%$ [17], whereas FaMe - Fall Management Exercise Intervention - has been shown to prevent falls by over $50 \%$ [18].

A variety of exercises focusing on lower limb muscles are generally included in fall prevention programmes, as they are important for functional movements and walking [17]. Ankle flexibility and strength, for example, are very important in this context, as they are associated with balance and balance recovery [17]. Exercises focusing on balance will help seniors not only to maintain balance but also to improve the capacity to recover balance in response to an external perturbation [17]. In this sense, training of stepping strategy as a response to avoid falls will have a huge impact on fall prevention [8]. Furthermore, exercises targeting the muscles which are the determinants of posture will have an impact on the compensatory strategies for balance control, being also an important part of a fall prevention programme [5].

To be effective, exercises have to be applied with the right duration and intensity to be sufficiently challenging and adapted for the individual [9], [17]. Progression is achieved by increasing exercises complexity and difficulty, adjusting speed of movements and duration [1]. Before starting to exercise, a warm-up phase is required to mobilize the joints and increase circulation, preparing the body for more vigorous exercise. After each session, cool-down is required to reduce body temperature and heart rate to pre-activity levels [5].

Exercises will improve functional capacity as well as seniors confidence in carrying out everyday activities without falling [9], [17]. They will also increase bone and muscle mass, preventing falls and fall-related fractures [5].

\section{RELATED WORK}

In recent years, the number of studies related to video games applied to the fields of health, rehabilitation and fall prevention has increased dramatically. Scientific community generally agree that exercise-based video games combining player movement, performance feedback and enjoyment can engage users to the game, promoting motivation and adherence to exercise programs [8], [6].

Dance Dance Revolution (DDR) is an example of a well known video game developed by Konami, in which players dance to respond to the step instructions given by the game [19]. This physical exercise was applied by Smith et al. [7] to verify the impact of a version of DDR on a regular stepping exercise program. For that they used a modified dance mat, needed to play DDR, that measured step timing and percentage of missed steps. Schoene, D. et al. (2013) has also explored DDR as a step training technology for fall prevention [8].

Another well-known project in which technology to support strength and balance training in older adults was developed is the iStoppFalls [1]. The solution uses Microsoft Kinect ${ }^{\circledR}$ to acquire movement data and a GoogleTV ${ }^{\mathrm{TM}}$ set-top box connected to the television [6]. The platform includes games for postural control, including walking, weight shifting, knee bending and stepping. In addition, cognitive tasks are added to the games to train dual tasking activities [1]. One of the variants of this project was proposed by Ejupi, A. et al. (2014) and consisted in implementing a game where the player does steps to the left or right according to the indications that appear on the television screen [2]. To that purpose, they combined Microsoft Kinect ${ }^{\circledR}$ with a 3D-accelerometer placed on the trunk to measure temporal and spatial parameters and to quantify the differences between fallers and non-fallers' performances [2].

SilverFit is another 3D-camera-based system that incorporates exercises mainly devoted to rehabilitation. However, a number of games included in the system were also proven useful for fall prevention, because they stimulate balance and stepping, also adding a cognitive component to the games [20]. Seniors who played the games found them challenging, fun and motivating [20].

Within the scope of rehabilitation, several systems based on the Microsoft Kinect ${ }^{\circledR}$ have been proposed [21]. Moreover, systems making use of inertial sensors and smartphones can be found, as is the case of UBI-Rehab [22]. In this system, multiple patients can play a collaborative game using an eGlove that captures hand motion during the practice of rehabilitation exercises.

Gardner, B. (2011) studied the effectiveness of Nintendo ${ }^{\circledR}$ Wii Fit ${ }^{\mathrm{TM}}$ as a motivating modality for fall prevention, and demonstrated its feasibility in improving balance, therefore reducing the risk of falling in older adults [23]. Bainbridge, E. et al. (2011) has also found that an intervention programme including the Wii Fit ${ }^{\mathrm{TM}}$ may be an effective low cost alternative to expensive force plate-based systems [24].

\section{SySTEM DEVELOPMENT}

One of the main issues in similar projects is their dependency on specific sensing technology, software platforms and hardware architectures. Technology is evolving fast and new solutions should provide modular design and open software architectures so that they can be up to date with emerging new technology without major effort. This project was designed having this in mind and provides a clear separation between higher application layers (interfaces, game logic and interactive components) and lower layers (sensor support and communication). Moreover, it uses Unity as software developing framework alongside some native plugins, allowing support for multiple platforms (desktop, mobile, web) while sharing the same code base. 


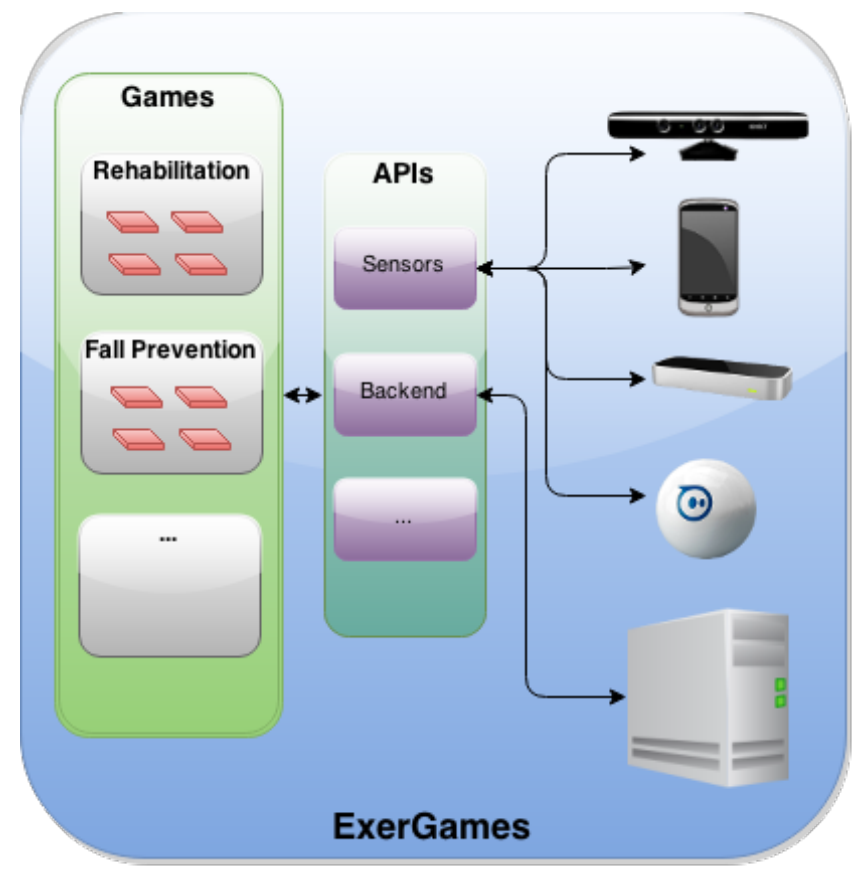

Fig. 1: ExerGames system architecture

\section{A. Architecture}

The application was designed to be easily expandable and start reaching other fields in the future, like focusing on cognitive impairments. Therefore, the software architecture is based on a three-tier framework that organizes modules (games) into dynamic categories, acting as a launcher and providing them a set of APIs for common functionalities like sensor communication, user profile and game session management (Fig. 1).

This architecture allows the modules to be agnostic on which sensors are currently supported and being used, as well as which backend server may be used to manage user profiles and store game session data. The API for sensor communication provided by the framework is implemented as shared library/DLL in pure native code $(\mathrm{C}++)$ and supports most of the existing platforms: Windows, Linux and Android. It deals with all communication details with any sensor, even when running everything locally on a mobile device. All data received from sensors is then processed and merged into a single protocol and send to any client (game) that asked for it and provided a callback. The protocol considers data from inertial sensors, gestures, body tracking, hand tracking and finger tracking. The framework itself is a client for sensor data, as it implements a sensor fusion algorithm that allows estimating the orientation of a connected smartphone and use it as a pointer to control the whole application. Using Unity to develop the project not only streamlines the development of the application, namely in what it takes to the user interfaces and interactive components, but also allows easy support for different platforms: desktop, mobile and web.

\section{B. Sensors}

The main motivation behind this project is to be used in community or domestic unsupervised contexts, using standard and relatively inexpensive equipment. Therefore, currently there is support for a small set of available sensor devices like smartphones (with their inertial sensors), Microsoft Kinect ${ }^{\circledR}$ (or similar depth sensors), Orbotix Sphero ${ }^{\circledR}$ and Leap Motion ${ }^{\circledR}$. The support for each sensor is dependent on the platform on which the application is running. Thus, all sensors are supported in desktop platforms, while depth sensors and Leap Motion ${ }^{\circledR}$ are still not supported on mobile. Several sensors (even of the same type) can be connected and used at the same time. The support for smartphones is done by running a small application on it that acts as a Bluetooth ${ }^{\circledR}$ server. It collects all data from available sensors (usually accelerometer, gyroscope and magnetometer) at a specific rate, eventually sending it to the games running on the other endpoint of the Bluetooth ${ }^{\circledR}$ link. By using the sensor fusion algorithm integrated in the ExerGames application to compute the smartphone orientation, alongside the minimal user interface to get click events, the user may control the system by using the smartphone also as a pointing device.

\section{Physical Rehabilitation}

The initial motivation of this project started with the idea of developing interactive games to help stroke victims in the rehabilitation process. A few simple games were developed to improve upper limbs movements in hemiparetic patients, while using Kinect ${ }^{\circledR}$ to track the exercises and collect important parameters like minimum and maximum angles between the arm and forearm and the arm and torso. Other games were further developed to help in the rehabilitation of the hand by using the Leap Motion ${ }^{\circledR}$ and Orbotix Sphero ${ }^{\circledR}$ sensors.

\section{Fall Prevention}

A literature review was performed to collect evidence and select a set of exercises specific for older adults with proven efficacy in their ability to improve balance, flexibility, mobility, gait, strength, muscle endurance and reaction time. Physical instructors and physiotherapists were involved since the beginning of the project, helping in defining exercises and maximizing their role in fall prevention. Authors had also the opportunity to actually observe seniors performing exercises to better understand their difficulties and what they enjoyed the most. Exercises were then organized in workouts (currently three are forecasted), i.e. exercise sessions, with a total duration of about 30 to 40 minutes. Each workout features exercises for the core elements of a fall prevention programme, i.e. lower limb strength, ankle stretch and strength, posture and balance. Warm-up and cool-down exercises were adapted to the muscular groups involved in the workout.

Whenever possible, exercises were presented to the subject as games, and a smartphone was used to monitor exercise performance. The rest of the exercises are presented to the user as an instructional video. Algorithms were developed to analyse smartphone sensor data and evaluate exercise movements.

At this stage, a total of four exercises were implemented and tested, including stepping on the same spot, standing in one leg, elevation of the upper limbs and sit to stand. The smartphone was used in the pocket, or, in case exercises were devoted to the upper limbs, it was used in the hand. 
Sensor fusion algorithm output was used to analyse the movement of the person while playing games, by means of detecting changes in smartphone orientation during the game, which were related to the movements performed. For stepping analysis, for example, it was considered that the angular movement produced by the legs during the exercise could be captured by the smartphone in the pocket. With leg movements, extreme thigh orientations would be detected, enabling the algorithm to count steps. Similarly, sit to stand exercise and one leg standing were based on the same basis that leg movements are captured by the smartphone in the pocket. Regarding the upper limb elevation exercise, smartphone had to be secured in the hand, so that the smartphone would follow and capture arm movements.

For each exercise a different single player game was created. A multiplayer game was also created and used to probe the dynamics of multiplayer gaming. This resulted in a total of five games:

1) Walk in the City (Fig. 2a): To play the game, the user performs steps on the same spot and controls an avatar walking through a city. From time to time an obstacle is shown and the player has to stop to avoid it. This game will not only promote the stepping exercise, but also add a challenging and recreational component. At the end of the exercise, it is possible to retrieve some metrics like number of steps or average cadence.

2) Fruit Match (Fig. 2b): The sit to stand movement is used to create a selection game. The user has to select if the fruit displayed on a side of the screen is matching or not a shape of a shadow displayed on the other side. If the user is sitting down, his answer is registered as no and if the user is standing up the answer is registered as yes. The game will promote the execution of sit to stand exercise, stimulating a certain amount of repetitions. At the end of the exercise, it is possible to retrieve metrics like the number of repetitions and ratio of sit-to-stand and stand-to-sit movements duration.

3) Hungry Cat (Fig. 2c): This game stimulates the elevation of the upper limbs. It consists of a user controlling a sitting avatar holding an aquarium with his hands and a cat that once in a while tries to catch the fishes on the aquarium. The goal of the game is to lift the aquarium as much as possible not letting the cat get the fish. At the end of the exercise, it is possible to retrieve metrics like the total amount of time spent with hands up and the number of repetitions.

4) Balance (Fig. 2d): In this game, the user controls an avatar that tries to balance a ball with his leg without letting it fall. To do that, the user has to maintain his/her leg raised for a certain amount of time. If the leg drops below a predefined threshold, the ball falls. In the background, movement amplitude and players' maximum balance time are measured.

5) The Horse Race (Fig. 2e): This is a multiplayer game in which the exercise of stepping on the same spot is used to simulate a race between two users. The idea here is having each user controlling a different horse jockey (each user carrying a smartphone) on a horse race and the user that does more steps during the race time wins.

The metrics retrieved during each game session can be saved in a backend server and attached to the user profile for further analysis.

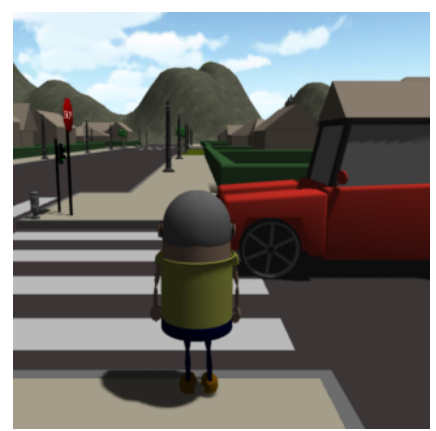

(a) Walk in the City game

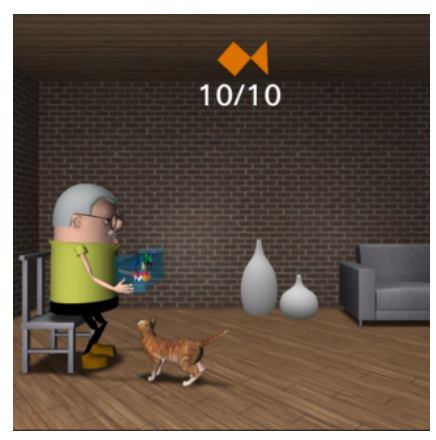

(c) Hungry Cat game

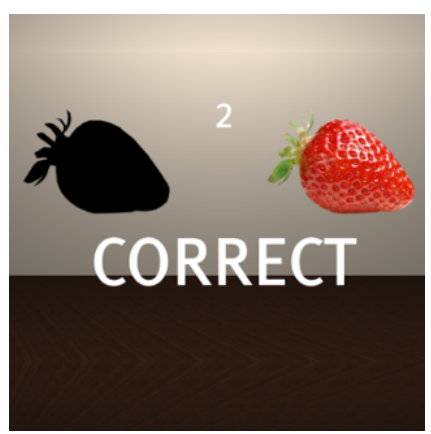

(b) Fruit Match game

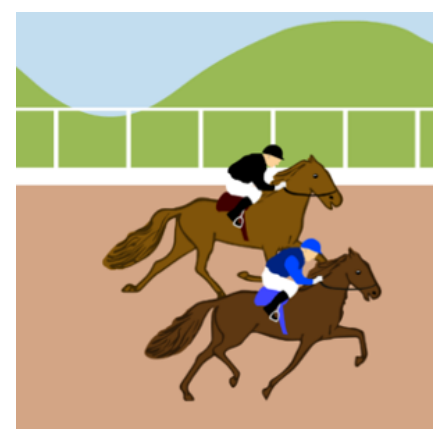

(e) The Horse Race game

Fig. 2: Screenshots of Fall Prevention games

\section{Testing Procedures}

A preliminary set of tests was conducted with older adults at this stage to get the first insights about the system.

Tests aimed mainly at evaluating whether older adults could or not interact successfully with the developed application. To do that, three critical factors were evaluated, including the capacity to use the smartphone as a pointer device, the usability of the current application's menus and the gameplay of each game. Simultaneously, data from the sensors and video were recorded to draw some conclusions about the performance of the developed algorithms in the evaluation of movement and game performance. It must be mentioned that the currently available games are only played with the smartphone (although navigation can be done with other sensors), but more are planned that will include the use of other sensors like the Kinect and the Wii Balance Board. However, tests on using the Kinect in the rehabilitation module of this solution are 
presented in [16].

\section{A. Participants}

A group of five volunteers (mean age $68.6 \pm 4.0$ years), four women and one man, living independently in their own housing, participated in the study. The subjects were selected randomly, but only those who were able to walk independently were considered for participation. Written informed consent was obtained from all participants prior to data collection.

\section{B. Protocol}

Participants were asked to perform eight predetermined tasks that were carefully designed to test two different aspects of the system, the overall usability and the usability of each of the developed games. The first three tasks focused on the ability of using the smartphone as a remote controller, and the overall navigability of the menus. The last five tasks focused on each individual game:

- Walk in the City game in which the user had to take around 100 steps on the same spot to follow a virtual path of nearly 60 meters;

- $\quad$ Fruit Match game in which the user had to complete 10 successful fruit matches (10 sit and stand movements);

- Hungry Cat game in which the participant had to hold his hands up three times for about 10 seconds each over a two minutes time period;

- Balance game in which the participant had to hold his leg up during 20 seconds;

- Horse Race game which lasted for one minute.

For each of these tasks the facilitator first briefed the participant about the game mechanics as well as its objectives and then asked the participant to play the game as they were told. Moreover, the participants were expected to be independent during the test as the facilitator was only expected to brief the users about the tasks at hand and move the smartphone's location (from pocket to hand and vice-versa) according to the game to be played.

Additionally, while participants were playing the games, smartphone sensor data were recorded. Each session was also recorded in video, to validate some results provided by movement analysis algorithms and evaluate the usability of the system.

\section{Evaluation}

System usability was evaluated by a quantitative and a qualitative analysis of the results. For the quantitative analysis, several measures were taken into account, including the number of tasks successfully executed by the participant and the number of errors done in each task. As an error, we considered an action that the user did not perform as expected such as not holding the phone properly during a specific exercise or selecting a wrong button on the user interface.

For the qualitative analysis, observations were detailed, including the tasks where the participants were the most comfortable with, what were the main difficulties while interacting with the system and what was the overall acceptance of the system by the users.

Movement evaluation metrics (i.e. number of repetitions, movements duration and amplitude) and game metrics (number of correct and incorrect movements) collected by the smartphone were compared with the metrics extracted from video recordings, for an initial validation of algorithms with real users in real usage scenarios.

\section{RESUlts}

Regarding the overall usability of the system, it was found that it was fairly easy for the participants to use the smartphone as a remote pointing device and to navigate through the menus. They were able to use the smartphone to point to the right spot on the screen without any difficulty, however, four out of five participants struggled with the selection process, which requires the user to do a single tap on the screen of the smartphone (shorter than half a second). A long press is used to enable/disable the smartphone as a pointing device. Some participants were not aware of the tapping events commonly used on smartphones and therefore they were frequently activating the enable/disable function.

All participants were able to finish all tasks (average of $0.64 \pm 0.14$ errors per task), however, some games could not be played properly. In general, participants performed well on all five games and most of the observed mistakes were due to some inaccuracy of the algorithms registering their movements and not participant's fault. This was particularly noticeable on the Walking in the City game that, despite the participants have understood its simple objective of stepping on the same place, they were not able to stop in time when they saw an obstacle, as the algorithm registered steps while they were not effectively taking them. The number of steps detected by the algorithm was in general highly above the real number of steps performed and captured in video (a total of 1014 steps instead of 430 steps were detected by the algorithm).

On the Fruit Match game (sit to stand exercise), only one participant finished the game despite one other participant had successfully played it at first, but also ended giving up on it due to the problems observed in movement detection. In fact, the smartphone could detect the sit to stand exercise of only one person accurately (10 out of 10 stands detected). The other tests results were discarded because the algorithm was not detecting movements related to this exercise. It was found later that an implementation error was present in this test that led to these poor results. In fact, the initial position was set while the person was still moving the smartphone before the game started, therefore, the reference for the sitting position was inappropriately set at the beginning. Nevertheless, all participants understood well the game and tried to play it correctly.

Three out of five participants played the Hungry Cat game (upper limbs elevation exercise) without any mistake. The other two participants had some difficulty at the beginning of the exercise in holding correctly the smartphone, but, after the help of the facilitator, they easily corrected the mistake. As the smartphone position changed during the game, arm movements were not correctly evaluated in these tests. On the other tests, 
a total of 11 elevations of the upper limbs (out of 11) were detected by the smartphone.

Finally, the Balance game (one leg standing) and the Horse Race game (stepping) were the games with the best results, as the participants did not make any mistake while playing them, even though one participant could not complete the Balance game due to a failure in movement evaluation. For the other samples, leg movements were captured and maximum time in one leg standing was accurately measured (relative errors close to $0 \%$ ).

\section{DISCUSSION}

Some problems were found in the evaluation of movements using the smartphone. In general, it was found that trousers' front pockets had multiple morphologies and sizes, which limited the accuracy of the algorithms in the detection of movement. In fact, it was expected that the smartphone orientation would change together with leg movements, therefore capturing solely the rotations of the thigh while performing the exercises. However, some users had their front pockets positioned above the thigh, meaning that the smartphone in this kind of pockets did not change its orientation together with leg movements. Others, on the other side, had very large pockets which led to changes in the position of the smartphone while moving the leg; in these specific cases, the smartphone was capturing not only leg movements but also the movements performed by itself inside the pocket.

According to the results and to the observations made, alternatives are required to improve movement detection. In case the smartphone cannot follow the changes in orientation of the thigh, the impacts created on the smartphone when the foot contacts with the ground could be used to detect steps.

Another possibility would be the use of an external band placed around the thigh, similar to those bands used to attach the smartphone to the arm. By using this accessory, it would be guaranteed that the smartphone would be capturing solely the movements of the thigh while exercising.

It may also be considered the use of complementary sensors, for example, an external accelerometer could be attached to the shoe to detect steps. Accelerometer could also be placed on the trunk to evaluate the sit to stand movement. Other sensors, like Microsoft Kinect ${ }^{\circledR}$ could be used in combination with the smartphone to improve movement detection and evaluation by adding additional information.

Regarding the upper limb elevation, it is required that the person is instructed to not change smartphone position while doing the exercise. As an alternative, a smartwatch attached to the arm could be used, preventing the sensors to measure other changes in orientation besides the rotation of the arm.

An important limitation of this approach, regardless the type of exercise conducted, is that the smartphone can only measure one body segment at each time. As a consequence, only right or left steps and arm movements can be evaluated, therefore, it is not guaranteed that the exercise is being performed exactly as expected. However, a person who is committed with the exercise and is aware of its role in fall prevention, he/she will try to follow the instructions and perform natural movements, without intention to fake the system.

Despite of the limitations evaluating the movements using the smartphone and the gender imbalance in the sample, all five participants that tested the system showed great enthusiasm and motivation while using it. They were able to successfully interact with it with almost no problem, besides some frustration caused by the difficulty positioning the smartphone correctly and the subsequent lack of accuracy of the system. Even when confronted with the visible lack of response of the system in the games that required the smartphone to be correctly placed in the pocket, some tried to came up with new approaches to get the games working like holding the smartphone against the leg by hand or asking for bands to hold it to their legs. Furthermore, some games still need improvements like the Fruit Match game that despite being easy to get a grasp off, it was reported by the users as a boring game contrasting with the others like the Horse Race game that really got them enthusiastic.

\section{CONCLUSION AND FUTURE WORK}

The topic of fall prevention is delicate due to the lack of pervasiveness factor in the existing solutions and in the inherent difficulty in validating the results. Preventive solutions results are always dependent on long trials and on a hard to measure effectiveness of a preventive action. One of the main drives for the development of this system was to allow being used in any context, without needing specific and unaffordable equipment. Therefore, the focus on using the smartphones as the main sensor device proved to be feasible if the movement detection algorithms are sufficiently accurate. During the tests that were made, it was clear that most of the algorithms were already accurate enough while others need a bit of work. This is now considered as future work and on top of priorities. Also, the ultimate goal is to have all exercises of the workouts as games, including the ones that are currently just displayed as an instructional video. Some of them will probably be possible with the help of a new sensor, a force platform (Wii Balance Board ${ }^{\mathrm{TM}}$ or similar), that is scheduled to be supported in the near future. Some others will certainly need the help of Kinect ${ }^{\circledR}$, which are already being developed. Using the concept of games proved to be effective in introducing this type of solutions in the daily routine, as it was clear the users' joy in performing the exercises and their willingness to play them again. However, the system is still lacking the support for different difficulty levels, which should be adapted to the user's functional status and add a progressive factor so the user may be challenged at every stage.

Acknowledgments: The authors would like to thank Dra. Anabela Martins (physiotherapist), Cátia Rodrigues and Serafim Faria (physical instructors) for their support, and acknowledge the financial support from North Portugal Regional Operational Programme (ON.2 - O Novo Norte), Portuguese National Strategic Reference Framework (NSRF) and the European Regional Development Fund (ERDF) from European Union through the project "Fall Competence Center" NORTE07-0124-FEDER-000041. Finally, the authors would like to thank all the users that have tested the system and provided their unvaluable feedback. 


\section{REFERENCES}

[1] Y. J. Gschwind, S. Eichberg, H. R. Marston, A. Ejupi, H. d. Rosario, M. Kroll, M. Drobics, J. Annegarn, R. Wieching, S. R. Lord, K. Aal, and K. Delbaere, "ICT-based system to predict and prevent falls (iStoppFalls): study protocol for an international multicenter randomized controlled trial," BMC Geriatrics, vol. 14, no. 1, p. 91, Aug. 2014.

[2] A. Ejupi, M. Brodie, Y. J. Gschwind, D. Schoene, S. Lord, and K. Delbaere, "Choice stepping reaction time test using exergame technology for fall risk assessment in older people," in Engineering in Medicine and Biology Society (EMBC), 2014 36th Annual International Conference of the IEEE, 2014.

[3] M. B. King, "Falls," in Hazzard's Geriatric Medicine \& Gerontology, 6th ed. McGraw-Hill, 2005, pp. 659-669.

[4] M. M. Gardner, D. M. Buchner, M. C. Robertson, and A. J. Campbell, "Practical implementation of an exercise-based falls prevention programme," Age and ageing, vol. 30, pp. 77-83, 2001.

[5] D. Skelton and S. Dinan, "Exercise for falls management: Rationale for an exercise programme aimed at reducing postural instability," Physiotherapy Theory and Practice, vol. 15, pp. 105-120, 1999.

[6] K. Aal, C. Ogonowski, T. von Rekowski, R. Wieching, and V. Wulf, "A fall preventive iTV solution for older adults," in TVX'14, 2014.

[7] S. T. Smith, C. Sherrington, S. Studenski, D. Schoene, and S. R. Lord, "A novel dance dance revolution (DDR) system for in-home training of stepping ability: basic parameters of system use by older adults," British journal of sports medicine, vol. 45, pp. 441-445, 2011.

[8] D. Schoene, S. R. Lord, K. Delbaere, C. Severino, T. A. Davies, and S. T. Smith, "A randomized controlled pilot study of home-based step training in older people using videogame technology," PloS One, vol. 8, no. 3, 2013.

[9] A. Charters, "Falls prevention exercise - following the evidence," Jun. 2013.

[10] L. A. Hale, "Community-based or home-based stroke rehabilitation: confusion or common sense?" New Zealand Journal of Physiotherapy, vol. 32, pp. 131-139, 2004.

[11] C. Ferreira, V. Guimarães, A. Santos, and I. Sousa, "Gamification of stroke rehabilitation exercises using a smartphone," in Proceedings of the 8th International Conference on Pervasive Computing Technologies for Healthcare. ICST (Institute for Computer Sciences, SocialInformatics and Telecommunications Engineering), 2014, pp. 282-285.

[12] H. R. Marston and S. T. Smith, "Interactive videogame technologies to support independence in the elderly: A narrative review," Games for Health Journal, vol. 1, no. 2, pp. 139-152, Apr. 2012.

[13] Microsoft, "Kinect," http://www.microsoft.com/en-us/ kinectforwindows/, 022014

[14] L. Motion, "Leap motion," http://www.leapmotion.com, 022014.

[15] Orbotix, "Sphero," http://www.gosphero.com, 022014.

[16] N. Matos, A. Santos, and A. Vasconcelos, "Kinteract: A multi-sensor physical rehabilitation solution based on interactive games," in Proceedings of the 8th International Conference on Pervasive Computing Technologies for Healthcare (PervasiveHealth '14). ICST (Institute for Computer Sciences, Social-Informatics and Telecommunications Engineering), 2014, pp. 350-353.

[17] J. Campbell and C. Robertson, "Otago exercise programme to prevent falls in older adults," 1997. [Online]. Available: http://www.acc.co.nz/PRD_EXT_CSMP/groups/external_ providers/documents/publications_promotion/prd_ctrb118334.pdf

[18] J. J., "Fitness and mobility exercise (FAME) program for stroke," Topics in geriatric rehabilitation, vol. 26, no. 4, pp. 310-323, 2010.

[19] Konami, "DDR," https://www.konami.com/ddr/, 2014.

[20] SilverFit, "SilverFit." [Online]. Available: http://www.silverfit.nl/index. php

[21] D. Webster and O. Celik, "Systematic review of Kinect applications in elderly care and stroke rehabilitation," J Neuroeng Rehabil, vol. 23, p. 26, 2014.

[22] Y. Choi, "Ubi-REHAB: An android-based portable augmented reality stroke rehabilitation system using the eGlove for multiple participants," in 2011 International Conference on Virtual Rehabilitation (ICVR). IEEE, 2011, pp. 1-2.
[23] B. Gardner, "Effectiveness of the Nintendo Wii Fit games on the balance of a community-dwelling older adult in eastern north california," Ph.D. dissertation, East Carolina University, Faculty of the Department of Occupational Therapy, Jul. 2011.

[24] E. Bainbridge, S. Bevans, B. Keeley, and K. Oriel, "The effects of the Nintendo Wii Fit on community-dwelling older adults with perceived balance deficits: A pilot study," Physical \& Occupational Therapy in Geriatrics, vol. 29, no. 2, pp. 126-135, May 2011. 